\title{
REALITY OF THE ZEROS OF AN ENTIRE FUNCTION AND ITS DERIVATIVES \\ BY
}

\author{
SIMON HELLERSTEIN, ${ }^{1}$ LI-CHIEN SHEN ${ }^{2}$ AND JACK WILLIAMSON \\ Dedicated to George Pólya
}

\begin{abstract}
In 1914 Pólya raised the problem of classifying the entire functions which together with all their derivatives have only real zeros. In earlier work Hellerstein and Williamson settled this problem for entire functions which are real on the real axis. We complete the classification in all cases and show that it is sufficient to consider the function and its first two derivatives.
\end{abstract}

Introduction. In [12 and 13], G. Pólya posed the problem of classifying all entire functions which have, along with all their derivatives, only real zeros. He conjectured that such a function must have one of the following forms:

$$
\begin{gathered}
f(z)=A e^{B z}, \\
f(z)=A\left(e^{i c z}-e^{i d}\right), \\
f(z)=A z^{m} e^{-a z^{2}+b z} \prod\left(1-\frac{z}{a_{n}}\right) e^{z / a_{n}},
\end{gathered}
$$

where $A$ and $B$ are complex constants, $a \geqslant 0, b, c, d$ and the $a_{n}$ are real, $\Sigma 1 / a_{n}^{2}<\infty$ and $m$ is a nonnegative integer.

In [10], B. Ja. Levin and I. V. Ostrovskii showed that if $f$ is a real (i.e. real on the real axis) entire function with only real zeros satisfying the growth condition

$$
\limsup _{r \rightarrow \infty}\{\log \log M(r, f) / r \log r\}=+\infty,
$$

then the zeros $\left\{c_{n}\right\}$ of $f^{\prime \prime}$ satisfy

$$
\sum_{c_{n} \neq 0}\left|\operatorname{Im}\left(1 / c_{n}\right)\right|=+\infty
$$

In particular, $f^{\prime \prime}$ has infinitely many nonreal zeros.

Pólya's conjecture was completely affirmed for real entire functions by two of the present authors in [6 and 7], where the cases of functions of finite order, and of infinite order growth slower than that of $(0.4)$, were successfully treated. These results coupled with those of Levin and Ostrovskii showed that if $f, f^{\prime}$, and $f^{\prime \prime}$ have only real zeros, and $f$ is real entire, then $f$ is in the Laguerre-Pólya class, i.e. of the form (0.3).

Received by the editors August 14, 1981 and, in revised form, December 31, 1981

1980 Mathematics Subject Classification. Primary 30D30; Secondary 30D35.

'Partially supported by NSF Grant MCS- 8003108.

${ }^{2}$ Part of research towards $\mathrm{Ph}$. D. degree, University of Wisconsin. 
In this paper we affirm the general conjecture of Pólya in which no assumption of reality is imposed on the function. We term a meromorphic function "strictly nonreal" if it is not a constant multiple of a real meromorphic function. In view of the preceding remarks we need only consider the class of strictly nonreal entire functions. We remark that M. Alander showed in 1923 [1] that if $f$ is a strictly nonreal entire function of finite order for which $f, f^{\prime}$, and $f^{\prime \prime}$ have only real zeros then $f$ is of the form (0.1) or (0.2) (a detailed proof appears in [6]). The infinite order case has remained open until now. Partial results have been obtained by Edrei [3], Levin and Ostrovskii [10], Hellerstein [5], and Hellerstein and Yang [9].

The proof that we shall present treats the finite order case along with the infinite order case, so that we find no advantage in appealing to Allander's result. Indeed, our methods apply to strictly nonreal meromorphic functions which have only real poles. Our main result is given by

THEOREM 1. Let $f$ be a strictly nonreal meromorphic function having only real poles. Suppose $f, f^{\prime}$, and $f^{\prime \prime}$ have only real zeros. If $f$ is entire, then $f$ is of the form $(0.1),(0.2)$, or one of the two forms

$$
\begin{gathered}
f(z)=A \exp \left(e^{i(c z+d)}\right), \\
f(z)=A \exp \{K[i(c z+d)-\exp i(c z+d)]\},
\end{gathered}
$$

where $A$ is a complex constant, $c$ and $d$ are real and

$$
-\infty<K \leqslant-1 / 4 \text {. }
$$

If $f$ has at least one pole, then $f$ is one of the two forms

$$
\begin{gathered}
f(z)=A e^{-i(c z+d)} / \sin (c z+d), \\
f(z)=A \exp [-2 i(c z+d)-2 \exp 2 i(c z+d)] / \sin ^{2}(c z+d),
\end{gathered}
$$

where $A, c$, and $d$ are constants, $c$ and $d$ real,

It is not difficult to show that if $f$ has one of the above forms, then $f^{\prime \prime \prime}$ must have some nonreal zeros. Theorem 1, therefore, combined with the results of Hellerstein and Williamson, mentioned in our earlier discussion of the real entire case, implies the following strong form of Pólya's conjecture.

THEOREM 2. Let $f$ be an entire function which has, along with its first three derivatives, only real zeros. Then $f$ is of the form $(0.1),(0.2)$ or $(0.3)$.

That functions of the form (0.6) have, along with their first two derivatives, no nonreal zeros was remarked by Edrei [3]. Our analysis leads us naturally to Edrei's example as well as to the unexpected class (0.7) and to the conclusion that the functions of $(0.6)$ and $(0.7)$ are the only entire functions of infinite order with this property.

Theorem 1 coupled with the remark following it shows that there are no strictly nonreal, nonentire meromorphic functions $f$ having only real poles for which $f, f^{\prime}, f^{\prime \prime}, f^{\prime \prime \prime}$ have only real zeros, and essentially only two such $f$ for which $f, f^{\prime}, f^{\prime \prime}$ have only real zeros. We are unable to classify the real meromorphic $f$ with this latter property, even under the assumption that all the poles are real. Results due to Hellerstein and Williamson [8] and J. Rossi [14] show that if $f$ is the reciprocal of a 
real entire function with only real zeros and $f^{\prime}, f^{\prime \prime}$ have only real zeros then either $f(z)=e^{a z^{2}+b z+c}$ or $f(z)=(A z+B)^{-n} ; a \leqslant 0, A \neq 0, b, B$ and $c$ are real constants and $n$ is a positive integer.

1. Tsuji's value distribution theory and some results of Levin and Ostrovskii. In addition to the classical value distribution theory of Nevanlinna we shall also require the less familiar half-plane theory of Tsuji. For a function meromorphic in the upper half-plane Tsuji [16] introduced the functionals

$$
m_{0}(r, f)=\frac{1}{2 \pi} \int_{\sin ^{-1}\left(r^{-1}\right)}^{\pi-\sin ^{-1}\left(r^{-1}\right)} \log { }^{+}\left|f\left(r \sin \theta e^{i \theta}\right)\right| \frac{d \theta}{r \sin ^{2} \theta},
$$

and

$$
N_{0}(r, f)=\int_{1}^{r} \frac{n_{0}(t, \infty)}{t^{2}} d t=\sum_{1 \leqslant r_{k} \leqslant r \sin \varphi_{k}}\left(\frac{\sin \varphi_{k}}{r_{k}}-\frac{1}{r}\right),
$$

where $\left\{r_{k} e^{i \varphi_{k}}\right\}$ are the poles of $f(z)$ and $n_{0}(t, \infty)$ denotes the number of poles of $f$ in the region $\{|z-i t / 2| \leqslant t / 2,|z| \geqslant 1\}$. Then the corresponding characteristic function for $f$ is defined by

$$
T_{0}(r, f)=m_{0}(r, f)+N_{0}(r, f),
$$

which, as Tsuji showed, is an increasing function of $r$.

The first fundamental theorem in the Tsuji setting takes the form

$$
m_{0}\left(r, \frac{1}{f-a}\right)+N_{0}\left(r, \frac{1}{f-a}\right)=T_{0}(r, f)+O(1) \quad(r \rightarrow \infty) .
$$

The initial part of our proof is based on the work of Levin and Ostrovskii [10]. The Tsuji functionals are central to their proofs. As is often the case when applying Nevanlinna's value distribution theory, the average smallness of the logarithmic derivative plays an important role. In the Tsuji format Levin and Ostrovskii [10, Lemma C] establish the following analogue of Nevanlinna's classical lemma on the logarithmic derivative.

LEMMA A. If $f(z)$ is meromorphic in the half-plane $\operatorname{Im} z>0$, then

$$
m_{0}\left(r, f^{\prime} / f\right)=O\left[\log \left(r T_{0}(r, f)\right)\right],
$$

as $r \rightarrow \infty$, except possibly for a set of $r$-values of finite measure.

As Levin and Ostrovskii observe, one can obtain the following analogue of a result of Hayman [4] by using the Tsuji functionals in place of the Nevanlinna functionals in both the statement and proof of Hayman's theorem.

LEMMA B. If $f(z)$ is meromorphic in the half-plane $\operatorname{Im} z>0$ including a neighborhood of $z=0$, then for integral $l \geqslant 1$,

$$
\begin{aligned}
T_{0}(r, f) \leqslant & \left(2+\frac{1}{l}\right) N_{0}\left(r, \frac{1}{f}\right)+\left(2+\frac{2}{l}\right) N_{0}\left(r, \frac{1}{f^{(l)}-1}\right) \\
& +O\left[\log \left(r T_{0}(r, f)\right)\right],
\end{aligned}
$$

except possibly for a set of r-values of finite measure. 
As Levin and Ostrovskii show in the same work [10, p. 332], the Tsuji $m_{0}$-functional and the more common average taken on semicircles centered at the origin are related by

LEMMA C. If $f$ is meromorphic in $\operatorname{Im} z>0$, then

$$
\int_{R}^{\infty} \frac{m_{0 \pi}(r, f)}{r^{3}} d r \leqslant \int_{R}^{\infty} \frac{m_{0}(r, f)}{r^{2}} d r \quad(R \geqslant 1)
$$

where

$$
m_{0 \pi}(r, f)=\frac{1}{2 \pi} \int_{0}^{\pi} \log ^{+}\left|f\left(r e^{i \theta}\right)\right| d \theta .
$$

Following the terminology in [10], we call any sequence $\left\{a_{n}\right\}$ of complex numbers satisfying $\Sigma_{a_{n} \neq 0}\left|\operatorname{Im}\left(1 / a_{n}\right)\right|<\infty$, an $A$-set.

The following result is implicit in [10, p. 333]. Since the proof is brief, we include it here.

LEMMA D. Let $f(z)$ be meromorphic. If the zeros of $\mathrm{ff}^{\prime \prime}$ and the poles of $f$ form an A-set, then

$$
T_{0}\left(r, f^{\prime} / f\right)=O(\log r) .
$$

Proof. Put $F=f / f^{\prime}$. Then $F^{\prime}=1-f f^{\prime \prime} / f^{\prime 2}$. Since the zeros and poles of $f$ and the zeros of $f f^{\prime \prime}$ form $A$-sets, the same is true for the zeros of $F$ and the one values of $F^{\prime}$. From (1.2) and the definition of an $A$-set it follows that $N_{0}(r, 1 / F)$ and $N_{0}\left(r, 1 /\left(F^{\prime}-1\right)\right)$ are bounded. Applying Lemma B with $l=1$ to $F$, we have $T_{0}(r, F)=O(\log r)$, outside a set of finite measure. The monotonicity of $T_{0}$ shows that we have this relation for all $r$. The proof is completed by referring to the First Fundamental Theorem (1.4).

2. The main lemma. Our proof of Theorem 1 is based on the following

LEMMA 1. Let $f$ and $f^{\prime}$ be strictly nonreal meromorphic functions with all their poles (if any) on the real axis. Suppose $f, f^{\prime}$, and $f^{\prime \prime}$ have no nonreal zeros. Then $f(z)=\overline{f(\bar{z})} e^{i h(z)}, f^{\prime}(z)=\overline{f^{\prime}(\bar{z})} e^{i g(z)}$ and

$$
\begin{gathered}
\frac{f^{\prime}}{f}(z)=\frac{1}{2} \frac{h^{\prime}(z)}{\sin (a z+b)} e^{i(a z+b),} \\
\frac{f^{\prime \prime}}{f^{\prime}}(z)=\frac{1}{2} \frac{g^{\prime}(z)}{\sin (A z+B)} e^{i(A z+B),} \\
g^{\prime}(z)=h^{\prime}(z)+2 a, \\
\frac{h^{\prime \prime}}{h^{\prime}}(z)+\frac{1}{2} h^{\prime}(z)[\cot (a z+b)-\cot (A z+B)] \\
=a[\cot (a z+b)+\cot (A z+B)],
\end{gathered}
$$

where $h^{\prime}$ and $g^{\prime}$ are real entire functions of order at most one, having only real zeros; and $a, b, A$ and $B$ are real constants.

Proof. Since $f$ and $f^{\prime}$ have only real zeros and poles

$$
f(z)=\overline{f(\bar{z})} e^{i h(z)}
$$


and

$$
f^{\prime}(z)=\overline{f^{\prime}(\bar{z})} e^{i g(z)},
$$

where $h$ and $g$ are real entire functions.

From (2.5) we obtain

$$
\frac{f^{\prime}}{f}(z)=\overline{\frac{f^{\prime}}{f}(\bar{z})}+i h^{\prime}(z),
$$

and we observe that every zero of $f^{\prime} / f$ is also a zero of $h^{\prime}$.

Moreover, from (2.7), the fact that $h^{\prime}$ is entire, and the definitions of $m_{0}$ and $T_{0}$ we see that

$$
T_{0}\left(r, h^{\prime}\right)=m_{0}\left(r, h^{\prime}\right) \leqslant m_{0}\left(r, \frac{f^{\prime}}{f}\right)+m_{0}\left(r, \overline{\frac{f^{\prime}}{f}(\bar{z})}\right)+\log 2 .
$$

Since $f$ and $f^{\prime \prime}$ have only real zeros, the zeros of $f f^{\prime \prime}$ form an $A$-set, and it follows readily from $(2.8)$ and Lemma $D$ that

$$
m_{0}\left(r, h^{\prime}\right)=O(\log r) .
$$

Repeating the above argument with $z$ replaced by $-z$ gives

$$
m_{0}\left(r, h^{\prime}(-z)\right)=O(\log r) .
$$

From (2.9), (2.10) and Lemma $\mathrm{C}$ we deduce

$$
\int_{R}^{\infty} \frac{T\left(r, h^{\prime}\right)}{r^{3}} d r=\int_{R}^{\infty} \frac{m_{0 \pi}\left(r, h^{\prime}\right)+m_{0 \pi}\left(r, h^{\prime}(-z)\right)}{r^{3}} d r=O\left(\frac{\log R}{R}\right) .
$$

Recalling that $T\left(r, h^{\prime}\right)$ is a nondecreasing function of $r,(2.11)$ yields

$$
T\left(r, h^{\prime}\right)=O(r \log r),
$$

so that $h^{\prime}$ is of order at most one.

Since all the zeros of $f^{\prime} / f$ are zeros of $h^{\prime}$, the exponent of convergence of the zeros of $f^{\prime} / f$ cannot exceed one. Denote by $\pi_{1}$ the Hadamard canonical product of the zeros of $f^{\prime} / f$ and let

$$
G=\pi_{1} f / f^{\prime} \text {. }
$$

Then $G$ is entire and since $\pi_{1}$ is entire of order at most one, an easy estimate of $m_{0}\left(r, \pi_{1}\right)$ from the definition (1.1) shows that $T_{0}\left(r, \pi_{1}\right)=m_{0}\left(r, \pi_{1}\right)=O\left(r^{\varepsilon}\right)$ for any $\varepsilon>0$. Combining these facts with the First Fundamental Theorem (1.4) and Lemma $\mathrm{D},(2.13)$ gives us

$$
\begin{aligned}
m_{0}(r, G) & =T_{0}(r, G) \leqslant T_{0}\left(r, f / f^{\prime}\right)+T_{0}\left(r, \pi_{1}\right)+\log 2 \\
& \leqslant T_{0}\left(r, f^{\prime} / f\right)+T_{0}\left(r, \pi_{1}\right)+O(1) \\
& =O(\log r)+O\left(r^{\varepsilon}\right)=O\left(r^{\varepsilon}\right),
\end{aligned}
$$

for every $\varepsilon>0$. Thus the order of $G$ is at most one. Let $\pi_{2}$ denote the canonical product of the zeros of $G$. Then the Hadamard factorization of $G$ takes the form

$$
G(z)=\pi_{2}(z) e^{-(p z+q)},
$$

where $p$ and $q$ are constants. 
Substituting from (2.15) into (2.13) we get

$$
\frac{f^{\prime}}{f}(z)=\frac{\pi_{1}}{\pi_{2}}(z) e^{p z+q} .
$$

Since, by assumption, the zeros and poles of $f$ and $f^{\prime}$ are real, we also have

$$
\overline{\frac{\pi_{1}}{\pi_{2}}(\bar{z})}=\frac{\pi_{1}}{\pi_{2}}(z)
$$

Applying (2.16) and (2.17) in (2.7) gives

$$
\frac{\pi_{1}}{\pi_{2}}(z) e^{p z+q}=\frac{\pi_{1}}{\pi_{2}}(z) e^{\bar{p} z+\bar{q}}+i h^{\prime}(z) .
$$

Solving for $\pi_{1} / \pi_{2}$ in (2.18) and substituting into (2.16) we obtain (2.1) with $a=\operatorname{Im} p, b=\operatorname{Im} q$. That $h^{\prime}$ has only real zeros is immediate from (2.1) and the reality of the zeros of $f^{\prime}$.

We now remark that in order to obtain (2.1) it was sufficient to know that $f$ and $f^{\prime}$ have only real zeros and poles and that $T_{0}\left(r, f^{\prime} / f\right)=O(\log r)$. Since we already know from the hypothesis that $f^{\prime}$ and $f^{\prime \prime}$ have only real zeros and poles, a repetition of the preceding argument would lead to (2.2) provided that we knew that $T_{0}\left(r, f^{\prime \prime} / f^{\prime}\right)=O(\log r)$. To prove that this latter relation holds, we differentiate (2.1) logarithmically to get

$$
\frac{f^{\prime \prime}}{f^{\prime}}(z)=\frac{f^{\prime}}{f}(z)+\frac{h^{\prime \prime}}{h^{\prime}}(z)+i a-a \cot (a z+b) .
$$

Since $h^{\prime}$ and $\sin (a z+b)$ are entire functions of finite order with only real zeros, Lemma $\mathrm{A}$ implies that $T_{0}\left(r, h^{\prime \prime} / h^{\prime}\right)$ and $T_{0}(r, a \cot (a z+b))$ are both $O(\log r)$. Since this same bound holds for $T_{0}\left(r, f^{\prime} / f\right)$, it follows from (2.19) that

$$
T_{0}\left(r, f^{\prime \prime} / f^{\prime}\right)=O(\log r) \text {. }
$$

Having established (2.20), we may now assert that (2.2) holds for a suitable real entire function $g^{\prime}$ of order at most one, having only real zeros, and suitable real constants $A$ and $B$.

To prove (2.3) and (2.4), we differentiate (2.1) logarithmically and compare the result with (2.2) to get

$$
\begin{gathered}
\frac{h^{\prime \prime}}{h^{\prime}}(z)+\left(\frac{1}{2} h^{\prime}(z)-a\right) \cot (a z+b)+\left(\frac{1}{2} h^{\prime}(z)+a\right) i \\
=\frac{1}{2} g^{\prime}(z) \cot (A z+B)+\frac{i}{2} g^{\prime}(z) .
\end{gathered}
$$

Letting $z$ be real, we equate real and imaginary parts in (2.21) to obtain the desired conclusions. This completes the proof of Lemma 1.

We remark that it is possible that $f$ is strictly nonreal, but $f^{\prime}$ is not. In that case $f^{\prime \prime} / f^{\prime}$ is real on the real axis, and (2.1) and (2.19), which still hold, imply $h^{\prime}(z)=-2 a$. Substituting this in (2.1) and integrating, we see that $f$ is of the form (0.9).

3. Restrictions on the constants $a$ and $A$ in Lemma 1 and the possible forms of $f$. We proceed to show that the constants $a$ and $A$ in Lemma 1 are severely restricted. In fact, we shall prove that the only possible values of these constants are (1) $a=0$, 
(2) $A=0$, (3) $a / A=1,2$, or 3 . Once these restrictions are established we determine the possible forms of $f$ by a straightforward integration. Our proof of the limitations on $a$ and $A$ is accomplished in a sequence of lemmas.

We begin with the simplest case, $a=0$.

LEMMA 2. If $f$ satisfies the hypothesis of Lemma 1 and $a=0$ in (2.1), then

$$
f(z)=K_{1} e^{K_{2} z},
$$

where $K_{1}$ and $K_{2}$ are constants, $K_{2}$ nonreal.

Proof. With $a=0,(2.1)$ and (2.4) take the form

$$
\frac{f^{\prime}}{f}(z)=\frac{1}{2} \frac{h^{\prime}(z)}{\sin b} e^{i b}
$$

and

$$
\left(\frac{1}{h^{\prime}}\right)^{\prime}(z)=\frac{1}{2}[\cot b-\cot (A z+B)] .
$$

Consideration of the multiplicity of a possible pole for the two sides of (3.3) shows that neither side can have any poles, so that $A=0$ and $\left(1 / h^{\prime}\right)^{\prime}$ is constant. Since $h^{\prime}$ is entire, we must have $h^{\prime}=K$, a constant. Integrating (3.2) with $h^{\prime}=K$ gives (3.1).

We next consider the case $a \neq 0$ and $A=0$.

LEMMA 3. Suppose $f$ satisfies the hypothesis of Lemma 1 . If $a \neq 0$ in (2.1) and $A=0$ in (2.2), then for some constant $K$

$$
f(z)=K \sin (a z+b) e^{i(a z+b)} .
$$

Proof. If $A=0,(2.4)$ becomes

$$
\frac{h^{\prime \prime}}{h^{\prime}}+\frac{1}{2} h^{\prime}[\cot (a z+b)-\cot B]=a[\cot (a z+b)+\cot B] \text {. }
$$

Since $h^{\prime}$ is real entire with only real zeros of order at most one, consideration of the Hadamard factorization of $h^{\prime}$ shows that as $y \rightarrow \infty,\left|\left(h^{\prime \prime} / h^{\prime}\right)(i y)\right|=o(|y|)$ (cf. [6, Lemma 4]) and that if $h^{\prime}$ has at least one zero, $\left|h^{\prime}(i y)\right| \geqslant O(|y|)$. Since

$$
\cot (\text { aiy }+b) \rightarrow \pm i \text { as } y \rightarrow \infty
$$

and $\cot B$ is real, we see that (3.5) is impossible unless $h^{\prime}$ has no zeros. Since $h^{\prime}$ is real entire of order $\leqslant 1$ and is also zero free, $h^{\prime}(z)=e^{\alpha z+\beta}$, with $\alpha$ and $\beta$ real. Comparing residues in (3.5) we require that $h^{\prime}=2 a$ at each zero of $\sin (a z+b)$. Hence $\alpha=0$ and $h^{\prime}(z)=e^{\beta}=2 a$ is necessary for (3.5) to hold. Substituting into (2.1) for $h^{\prime}$ gives

$$
\frac{f^{\prime}}{f}(z)=\frac{a e^{i(a z+b)}}{\sin (a z+b)}=a \cot (a z+b)+a i .
$$

Integrating (3.6) we obtain (3.4).

Having disposed of the cases $a=0$ and $A=0$, we assume from this point on that $a \neq 0$ and $A \neq 0$. Since a real affine transformation on $z$ does not affect the hypothesis of Lemma 1 , it is sufficient to treat only the functions $f$ for which $A=1$ and $B=0$. We shall refer to such $f$ as "normalized". Since we have shown that if $a=0$ then $A=0$, we have $a \neq 0$ for normalized $f$. 
LEMMA 4. If $f$ satisfies the hypothesis of Lemma 1 and is normalized, then a is a positive integer.

Proof. We first show that $a>0$. Consider (2.4) with $A=1$ and $B=0$. Suppose $a<0$. Using the growth properties of $h^{\prime}(i y)$ and $\left(h^{\prime \prime} / h^{\prime}\right)(i y)$ together with the fact that $\cot ($ aiy $+b)-\cot (i y) \rightarrow 2 i$ as $y \rightarrow+\infty$ if $a<0$, while $\cot ($ aiy $+b)+\cot (i y)$ $\rightarrow 0$, we see that (2.4) is impossible. Since $a \neq 0$, we must have $a>0$.

To prove that $a$ is an integer we again take $A=1$ and $B=0$, and we divide by $h^{\prime}$ in (2.4). This gives us the linear first order differential equation in $1 / h^{\prime}$,

$$
\left(1 / h^{\prime}\right)^{\prime}+a[\cot (a z+b)+\cot z]\left(1 / h^{\prime}\right)=\frac{1}{2}[\cot (a z+b)-\cot z] .
$$

By the standard application of an integrating factor we find that for $0<\varepsilon<z<\pi$,

$$
\begin{gathered}
\left(1 / h^{\prime}(z)\right) \sin ^{a} z \sin (a z+b)-\left(1 / h^{\prime}(\varepsilon)\right) \sin ^{a} \varepsilon \sin (a \varepsilon+b) \\
=-\frac{1}{2} \int_{\varepsilon}^{z} \sin [(a-1) t+b] \sin ^{a-1} t d t .
\end{gathered}
$$

Let

$$
H(z)=\frac{\sin ^{a} z \sin (a z+b)}{h^{\prime}(z)}, \quad 0<\varepsilon<z<\pi .
$$

Since $a>0$ the integral in (3.8) has a finite limit as $\varepsilon \backslash 0$ or as $z \nearrow \pi$, so that $H(z)$ has a continuous extension to the closed interval $[0, \pi]$.

Now suppose $a$ is not an integer. Since $h^{\prime}$ is entire and $H$ has a finite limit as $\varepsilon \searrow 0$ or as $z \nearrow \pi$, it is easily seen that

$$
H(0)=H(\pi)=0 .
$$

From (3.8), (3.9), and (3.10), we obtain

$$
\int_{0}^{\pi} \sin [(a-1) t+b] \sin ^{a-1} t d t=0 .
$$

We next consider (3.7) on the interval $(\pi, 2 \pi)$, with $a>0$ and again nonintegral. Letting $\zeta=z-\pi$ and $\tilde{h}(\zeta)=h(\zeta+\pi)$, (3.7) becomes

$$
\left(\frac{1}{\tilde{h}^{\prime}}\right)^{\prime}+a[\cot (a \zeta+a \pi+b)+\cot \zeta] \frac{1}{\tilde{h}^{\prime}}=\frac{1}{2}[\cot (a \zeta+a \pi+b)-\cot \zeta] .
$$

Proceeding exactly as we did for the equation (3.7), with the obvious integrating factor on $(0, \pi)$, an identical reasoning leads to the integral relation

$$
\int_{0}^{\pi} \sin [(a-1) t+a \pi+b] \sin ^{a-1} t d t=0 .
$$

From (3.11) and (3.13) we obtain

$$
\sin (a \pi) \int_{0}^{\pi} \cos [(a-1) t+b] \sin ^{a-1} t d t=0 .
$$

To complete our proof, we appeal to the formula [11, p. 158] (originally due to Cauchy [2,pp. 41-89])

$$
\int_{0}^{\pi} \sin ^{(a-1)} t e^{i[(a-1) t+b]} d t=\frac{\pi}{2^{a-1}} e^{i[\pi(a-1) / 2+b]} \neq 0 .
$$


For (3.11) and (3.15) to hold, the real part of the integral in (3.15) cannot vanish, and (3.14) implies that $\sin a \pi=0$, i.e. $a$ is an integer.

LEMMA 5. If $f$ satisfies the hypothesis of Lemma 1, and is normalized, then a = 1,2, or 3.

Proof. By Lemma 4, $a$ is a positive integer. Returning to the equations (3.7)-(3.9), we again observe from (3.8) that $H$ has a finite limit as $\varepsilon \searrow 0$ or as $z \nearrow \pi$ and therefore extends continuously to $[0, \pi]$, but that (3.10) need not hold. From (3.8) and (3.9) we do have for $0 \leqslant z \leqslant \pi$,

$$
H(z)-H(0)=-\frac{1}{2} \int_{0}^{z} \sin [(a-1) t+b] \sin ^{a-1} t d t .
$$

Since $a$ is a positive integer, (3.16) shows that $H$ extends to an entire function, and (3.9) holds for all complex $z$. The relations (3.9) and (3.16) imply that $H$ is real entire of exponential type with only real zeros, so that $H$ is in the Laguerre-Pólya class (i.e. can be factored in the form (0.3)).

Suppose $a>2$. Then (3.16) implies that for each integral value of $n, H^{\prime}(n \pi)$ vanishes with multiplicity exceeding one. We proceed to show that $a=3$. By a classical theorem of Laguerre [15, p. 266] the zeros of $H^{\prime}$ which are not also zeros of $H$ are simple and are separated by the zeros of $H$. Therefore, if $a>2$,

$$
H(n \pi)=0, \quad n=0, \pm 1, \pm 2, \ldots .
$$

From (3.16), (3.17), and the formula of Cauchy (3.15) we deduce that

$$
0=H(\pi)=\frac{\pi}{2^{a-1}} \sin \left[\frac{\pi}{2}(a-1)+b\right] .
$$

Since $a>2$ is an integer (3.18) implies that

$$
b \equiv \begin{cases}\pi / 2 \bmod \pi & \text { for } a \text { even, } a \geqslant 4 \\ 0 \bmod \pi & \text { for } a \text { odd, } a \geqslant 3 .\end{cases}
$$

We first consider the case $a>2$ with $a$ odd. Then $H(0)=0$ and from (3.16) and (3.19) we have

$$
H(z)=\frac{-\cos b}{2} \int_{0}^{z} \sin ^{a-1} t \sin (a-1) t d t \quad(b \equiv 0 \bmod \pi) .
$$

With $a>2$ odd it is clear from (3.20) that $H$ is periodic of period $\pi$. By the theorem of Laguerre referred to above, the zeros of $H^{\prime}$ which are not also zeros of $H$ must interlace with the zeros of $H$. Now $H(n \pi)=0$ and (3.9) implies all other zeros of $H$ are located at zeros of $\sin a z$. In addition, (3.20) implies that $H^{\prime}$ has zeros at the zeros of $\sin z$ and $\sin (a-1) z$. Therefore, it follows that on the interval $J=\{z$ : $-\pi /(a-1) \leqslant z \leqslant(a-2) \pi /(a-1)\}$,

$$
H(j \pi / a)=0 \quad(j=0, j=2,3, \ldots, a-2),
$$

and that $H$ vanishes at no other points of $J$. Since $H$ is real entire of exponential type with only real zeros and periodic of period $\pi$, we deduce from (3.21) and the fact that $H$ has a zero of multiplicity $(a+1)$ at $z=0$, together with a simple growth argument that

(3.22) $H(z)=C \sin ^{a} z \sin a z / \sin \left(z+\frac{\pi}{a}\right) \sin \left(z-\frac{\pi}{a}\right) \quad(C \neq 0$ a real constant $)$. 
Substituting into (3.9) we obtain

$$
h^{\prime}(z)=K \sin \left(z+\frac{\pi}{a}\right) \sin \left(z-\frac{\pi}{a}\right) \quad(K \neq 0 \text { a real constant }) .
$$

With $a$ still odd, we now suppose further that $a>3$. Returning to (2.4) with $A=1, B=0$, and $b \equiv 0 \bmod \pi$, a calculation of residues at $z=j \pi / a$, with $j=$ $2,3, \ldots, a-2$, gives

$$
h^{\prime}(j \pi / a)=2 a \quad(j=2,3, \ldots, a-2) .
$$

However, from (3.23) we find that on the interval $0<z<\pi h^{\prime \prime}(z)=0$ only for $z=\pi / 2$, so that Rolle's theorem applied to $h^{\prime}(z)-2 a$ implies that (3.24) can only hold for at most two values of $j$. Hence $a \leqslant 5$. If $a=5$, explicit integration of (3.20) gives a function with a zero not among the zeros of $\sin 5 z$. Thus $a \neq 5$.

We remark that we may still have $a=3$, and that in this case, (3.19) still gives $b \equiv 0 \bmod \pi$.

For $a>2$ even, we proceed in a similar fashion. From (3.16), (3.17), and (3.19) we have

$$
H(z)=-\frac{1}{2} \sin b \int_{0}^{z} \sin ^{a-1} t \cos (a-1) t d t \quad\left(b \equiv \frac{\pi}{2} \bmod \pi\right) .
$$

Thus $H$ is periodic with period $\pi$. Since $H$ is real entire of exponential type and by (3.9) and (3.19) has all its zeros among the zeros of $\sin ^{a} z \cos a z, H$ is in the Laguerre-Pólya class. In this case we consider the real closed interval from $-\pi / 2 a$ to $(2 a-1) \pi / 2 a$. An argument analogous to that in the odd case shows that on $J, H$ vanishes exactly at the points $z=0$ and $z=-\pi / 2 a+j \pi / a, j=2,3, \ldots, a-1$, and that

$$
h^{\prime}(z)=K \sin \left(z+\frac{\pi}{2 a}\right) \sin \left(z-\frac{\pi}{2 a}\right) \quad(K \neq 0 \text { a real constant }) .
$$

Substituting from (3.26) into (2.4) with $a$ even, $b \equiv \pi / 2 \bmod \pi, A=1$ and $B=0$, and arguing as we did in the case when $a$ is odd, we conclude that if $a$ is even it cannot exceed 2.

\section{Proof of the theorems.}

Proof of Theorem 1. If $f$ is strictly nonreal, but $f^{\prime}$ is not, then by the remark made after the proof of Lemma $1, f$ is of the form (0.9). Thus in the remainder of the proof we shall assume that $f$ and $f^{\prime}$ are strictly nonreal so that Lemmas 1-5 hold. From Lemma 5 it follows that if $f$ satisfies the hypothesis of Theorem 1 and is normalized then in (2.1) $a=1,2$, or 3 . In all of these instances, with $H$ defined by (3.9), the considerations at the beginning of the proof of Lemma 5 show that $H$ is in the Laguerre-Pólya class with $H$ representable by (3.16).

If $a=3$, the subsequent argument in the proof of Lemma 5 shows that in (3.16) we also have $H(0)=0$ and $b \equiv 0 \bmod \pi$, so that upon integrating we obtain

$$
H(z)=\frac{-\cos b}{4} \sin ^{4} z \quad(b \equiv 0 \bmod \pi) .
$$

Substituting from (4.1) into (3.9) with $a=3$ gives

$$
h^{\prime}(z)=4 \sin 3 z / \sin z \text {. }
$$


Further substitution from (4.2) into (2.1), (2.3), and (2.2) with $a=3$, and $b \equiv 0$ $\bmod \pi, A=1$, and $B=0$ yields

$$
\frac{f^{\prime}}{f}(z)=-2 e^{3 i z} / \sin z
$$

and

$$
\frac{f^{\prime \prime}}{f^{\prime}}(z)=(3 \sin z-2 \sin 3 z) e^{i z} / \sin ^{2} z=(1-4 \cos 2 z) e^{i z} / \sin z
$$

From (4.3) and (4.4) we see that $f^{\prime}$ and $f^{\prime \prime}$ have only real zeros. Integration of (4.3) leads to

$$
f(z)=C e^{-2 i z} \exp \left(-2 e^{2 i z}\right) / \sin ^{2} z \quad(C \text { a complex constant }),
$$

which upon removing the normalization is equivalent to $(0.10)$.

If $a=1$ or 2, the proof of Lemma 5 gives no immediate information concerning the values of $H(0)$ and $b$ in (3.16). For each of these cases we begin with (3.16) and argue directly.

When $a=1$, we integrate (3.16) and obtain

$$
H(z)=-\frac{1}{2}(\sin b) z+H(0)=c_{1} z+c_{2},
$$

where $c_{1}$ and $c_{2}$ do not both vanish. Substituting from (4.6) into (3.9) with $a=1$, we find that

$$
h^{\prime}(z)=\frac{\sin z \sin (z+b)}{c_{1} z+c_{2}} .
$$

Recalling that $g^{\prime}$ defined by (2.2) has only real zeros, (2.3) together with (4.7) imply that

$$
G(z)=\sin z \sin (z+b)+2 c_{1} z+2 c_{2}
$$

has only real zeros. If $c_{1} \neq 0$, then $G$ would have only finitely many zeros, since $|G(z)| \rightarrow \infty$ as $|z| \rightarrow \infty$ along the real axis. But $G$ is of finite order so that it would also follow (e.g. from the Hadamard factorization of $G$ ) that $G^{\prime \prime}$ would have only finitely many zeros. However, $G^{\prime \prime}(z)=2 \cos (2 z+b)$ has infinitely many zeros. Thus $c_{1}=-\sin b=0$, and (4.7) becomes

$$
h^{\prime}(z)=c \sin ^{2} z \quad(c \neq 0 \text { a real constant }) .
$$

From (4.8) and (2.3) we now have

$$
g^{\prime}(z)=c \sin ^{2} z+2 \text {. }
$$

Since $g^{\prime}$ has only real zeros, (4.9) implies that

$$
c \leqslant-2 \text {. }
$$

Substituting from (4.8) into (2.1) with $a=1$ and $b \equiv 0 \bmod \pi$ and integrating, we obtain

$$
f(z)=C \exp \left\{\frac{c}{8}[2 i z-\exp (2 i z)]\right\}
$$

$(C \neq 0$ a complex constant and $c \leqslant-2)$. 
Allowing $z$ to undergo a real affine transformation, putting $K=c / 8$ and recalling (4.10), we find that $f$ is of the form (0.7) with $K$ subject to $(0.8)$.

When $a=2$, integrating (3.16) gives

$$
\begin{aligned}
H(z) & =\frac{1}{8} \sin (2 z+b)-\frac{1}{4}(\cos b) z-\frac{1}{8} \sin b+H(0) \\
& =\frac{1}{8} \sin (2 z+b)+c_{3} z+c_{4} .
\end{aligned}
$$

Since (3.9) implies that $H$ has only real zeros, an argument identical to the one used in the case $a=1$ to deduce that $c_{1}=0$ in (4.7) shows that $c_{3}=0$, so that $b \equiv \pi / 2(\bmod \pi)$. It follows then from $(3.9)$ and $(4.12)$ that

$$
h^{\prime}(z)=\frac{8 \sin ^{2} z \cos 2 z}{\cos 2 z+c} .
$$

In order that $h^{\prime}$ be entire the constant $c$ can only have the values 0 or -1 . If $c=0$, combining (4.13) and (2.3) with $a=2$ gives $g^{\prime}(z)=8 \sin ^{2} z+4$. Since the zeros of $g^{\prime}$ are required to be real, we cannot have $c=0$.

For $c=-1,(4.13)$ may be expressed as

$$
h^{\prime}(z)=-4 \cos 2 z \text {. }
$$

Substituting from (4.14) into (2.1) with $a=2$ and $b \equiv \pi / 2(\bmod \pi)$ we find that

$$
\frac{f^{\prime}}{f}(z)=-2 i e^{2 i z} \text {. }
$$

Integrating (4.15) we obtain

$$
f(z)=C \exp \left(-e^{2 i z}\right) \quad(C \text { a complex constant }),
$$

which, allowing for a real affine transformation, is equivalent to (0.6).

Recalling Lemmas 2 and 3, we may now conclude that if $f$ is strictly nonreal and $f, f^{\prime}, f^{\prime \prime}$ have only real zeros, then $f$ is of the form $(0.1),(0.2),(0.6)$ or $(0.7)$ if $f$ is entire, and that $f$ is of the form (0.9) or $(0.10)$ if $f$ is meromorphic but not entire and all its poles are real. This completes the proof of Theorem 1 .

Proof of Theorem 2. To see that Theorem 2 is a consequence of Theorem 1, we observe that if $f$ is of the form $(0.6)$ or $(0.7)$ then $f$, and hence $f^{\prime}$, is of infinite order. It then follows that $f^{\prime}$ is neither of the form $(0.6)$ or $(0.7)$. Thus we cannot have $f^{\prime}, f^{\prime \prime}, f^{\prime \prime \prime}$ with only real zeros if $f$ is strictly nonreal with no nonreal zeros unless $f$ is of the form $(0.1)$ or $(0.2)$. By direct consideration of $f^{\prime \prime \prime} / f^{\prime \prime}$ it may in fact be shown that $f^{\prime \prime \prime}$ has infinitely many nonreal zeros and no real zeros.

We remark that if $f$ is of the form (0.9), an easy computation shows that $f^{\prime \prime \prime}$ has only nonreal zeros. If $f$ is of the form (0.10), a computation shows that the poles of $f^{\prime}$ are of multiplicity three, so that $f^{\prime}$ is not of the form $(0.10)$. Thus, $f^{\prime \prime \prime}$ has nonreal zeros in this case also.

\section{REFERENCES}

1. M. Ålander, Sur les fonctions entières qui ont tous leurs zéros sur une droit, C. R. Acad. Sci. Paris Sér. A-B 176 (1923), 158-161.

2. A. L. Cauchy, Ouevres de Cauchy 2 ième série, Tom XV, Gauthier-Villars, Paris, 1974.

3. A. Edrei, Meromorphic functions with three radially distributed values, Trans. Amer. Math. Soc. 78 (1955), 276-293. 
4. W. K. Hayman, Picard values of meromorphic functions and their derivatives, Ann. of Math. (2) 70 (1959), 9-42.

5. S. Hellerstein, On the zeros of an entire function and its second derivative, Illinois J. Math. 19 (1966), 488-496.

$6 \mathrm{~S}$. Hellerstein and J. Williamson, Derivatives of entire functions and a question of Pólya, Trans. Amer. Math. Soc. 227 (1977), 227-249.

7. __ Derivatives of entire functions and a question of Pólya. II, Trans. Amer. Math. Soc. 234 (1977), 497-503.

8. The zeros of the second derivative of the reciprocal of an entire function, Trans. Amer. Math. Soc. 263 (1981), 501-513.

9. S. Hellerstein and C. C. Yang, Half-plane Tumura-Clunie theorems and the real zeros of successive derivatives, J. London Math. Soc. (2) 4 (1972), 469-481.

10. B. Ja. Levin and I. V. Ostrovskii, On the dependence of the growth of an entire function on the distribution of the zeros of its derivatives, Amer. Math. Soc. Transl. (2) 32 (1963), 323-357.

11. N. Nielsen, Handbuch der Theorie der Gammafunktion, Teubner, Leipzig, 1906; reprinted in Die Gammafunktion, Chelsea, New York, 1964.

12. G. Pólya, Sur une question concernant les fonctions entières, C. R. Acad. Sci. Paris Ser. A-B 158 (1914), 330-333.

13. , Bemerkung zur theorie der ganzen Funktionen, Jahresber. Deutsch. Math.-Verein. 24 (1915), $392-400$

14. J. Rossi, The reciprocal of an entire function of infinite order and the distribution of the zeros of its second derivative, Trans. Amer. Math. Soc. 270 (1982), 667-683.

15. E. C. Titchmarsh, The theory of functions, 2nd ed., Oxford Univ. Press, London, 1939.

16. M. Tsuji, On Borel's directions of meromorphic functions of finite order. I, Tôhoku Math. J. (2) 2 (1950), 97-112.

Department of Mathematics, University of Wisconsin, Madison, Wisconsin 53706

Department of Mathematics, University of Hawail, Honolulu, Hawail 96822 